\title{
Assessment of symptom severity in chronic rhinosinusitis with and without polyps- a prospective study in tertiary care centre at Mandya
}

\author{
Balaji N.K. ${ }^{1}$, Ravi D. ${ }^{2}$, Prasad MH. ${ }^{3}$ \\ ${ }^{1}$ Dr. Nagavara Kalegowda Balaji, Assistant Professor, ${ }^{2}$ Dr. Ravi D, Assosiate Professor, ${ }^{3}$ Dr. MH Prasad, Professor; all \\ authors are attached with Department of ENT, Mandya Institute of Medical Sciences, Mandya, Karnataka, India.
}

Corresponding Author: Dr. Nagavara Kalegowda Balaji, Assistant Professor, Dept of ENT, MIMS, Mandya, Karnataka, India, Address- First Cross, Ashok Nagar, Mandya, Karnataka, India. E-mail- drnkbalaji@gmail.com

\begin{abstract}
Chronic Rhinosinusitis has been classified into Chronic Rhinosinusitis with Polyps (CRSwNP) and Chronic Rhinosinusitis without Polyps (CRSsNP) in accordance with the manifestation of the chronic inflammatory process affecting the Sinonasal mucosa. Two subtypes of CRS have varied clinical and radiologic manifestations. Wide range of symptoms and their severity significanty affects the quality of living which can be systematically assessed using QOL questionnaire. Objectives: To assess the symptoms severity using SNOT 22 questionairre in CRSwNP and CRSsNP. Using the above data, the possibility of diagnosis of presence or absence of nasal polyps by symptom analysis will be correlated Materials and Methods: Duration of the study was two years from May 2016 to April 2018 and Convenient (purposive)sampling method was used for sampling. SNOT 22 sino nasal outcome test questionnaire, a validated tool was used to assess the patients. All these patients were evaluated according to the standard laboratory profiles of allergy. Analysis Data was entered into Microsoft Excel (Windows 7; Version 2007) and analyses were done using the Statistical Package for Social Sciences (SPSS) for Windows software (version 22.0; SPSS Inc, Chicago). Results: While Nasal obstruction was the most important symptom, Smoking tobacco(cigarettes) was also the most important characteristic that significantly affected both groups. Headache, Facial pain and Pressure were also universally present in both groups. Seromucinous Rhinorrhoea (4.03 vs 1.79; $\mathrm{p}$ value 0.001 ), Sneezing (4.24 vs 2.35; p value 0.001), Alteration of Taste and Smell (3.64 vs 1.25; p value 0.001) were also the factors that affected the quality of life. Conclusion: There is a considerable overlap in the presentation and no actual correlation can be made by using only symptomatology as a tool. Endoscopic and CT scan findings are the most important entities to finally differentiate these two conditions along with immunological studies.
\end{abstract}

Keywords: CRSwNP, SNOT 22, Quality of Life

\section{Introduction}

Chronic Rhinosinusitis (CRS) is a significant health burden with an prevalence of $10.9 \%$ in Europe, $4.5-12 \%$ in the United States and $6.8 \%$ in Asia. The diagnosis of CRS is based on well-defined criteria which include a combination of specific symptoms and signs, confirmed with endoscopic and radiological findings.

Chronic Rhinosinusitis is defined according to European position paper on Rhinosinusitis and Nasal polyposis (EPOS) as an inflammation of the Nose and Paranasal Sinus mucosa characterised by two or more symptoms lasting for more than 12 weeks like Nasal obstruction, Nasal discharge supplemented with Facial

Manuscript received: $26^{\text {th }}$ July 2019

Reviewed: $7^{\text {th }}$ August 2019

Author Corrected: $16^{\text {th }}$ August 2019

Accepted for Publication: $21^{\text {st }}$ August 2019 pain or pressure, reduction or loss of smell along with endoscopic and CT scan findings. Two broad classifications exist, viz., chronic rhinosinusitis with polyps (CRSwNP) and chronic rhinosinusitis without nasal polyps (CRSsNP). The treatment of CRS involves an initial course of maximal appropriate medical therapy followed by surgery in patient's refractory to medical treatment $[1,2,3]$.

In accordance with the manifestation of the chronic inflammatory process affecting the Sinonasal mucosa, nasal polyposis has higher inflammatory reaction with IgE, IL-5, Eosinophilia as compared to non-polyposis. Response to treatment also varies with CRSwNP responding to anti-inflammatory agents like Topical and Systemic Steroids better with almost absolute reversal 


\section{Original Research Article}

of early disease. CRSsNP is a resistant disease with a different morbidity profile $[1,2]$.

Sinonasal outcome test 22 is a standard questionnaire which has been used to assess the outcome of medical and surgical management of Chronic Rhinosinusitis with and without polyposis.

The questionnaire contains Rhinologic symptoms and Psychological domains which are commonly seen patients suffering from Chronic Rhinosinusitis.

In this study, the SNOT 22 was used to compare the symptom severity in the two phenotypes of CRS for the presence or absence of polyps $[1,2]$.

\section{Materials and Methods}

Study Setting, Duration, Type: The study is a Prospective Cross-sectional design for Two years from May 2016 to April 2018 at Dept of ENT, Mandya Institute of Medical Sciences, Mandya

Ethical consideration: The study was undertaken after obtaining permission from the Institutional Ethics Committee

\section{Inclusion Criteria}

1. Patients with symptoms of Chronic Rhinosinusitis according to EPOS criteria

2. Patients aged above 20 years

\section{Exclusion criteria}

1. Patients with Symptoms with Chronic Rhinosinusitis with other significant comorbidities that can alter the course of CRS like Granulomatous diseases, Autoimmune diseases, Malignacies etc.

\section{Patients below 20 years}

\section{Patients of CRS refusing to consent for the study}

Sampling: Convenient sampling (Purposive)was employed since the study is Questionnaire based.

Sample size was 230 which was corrected for statistical purposes as 231 .

Selection was on EPOS criteria which underwent further division into CRSwNP and CRSsNP according to the procedure.
Method of collection of data, procedure- Patients visiting ENT outpatient department of Mandya Institute of Medical Sciences with major and minor symptoms of Chronic Rhinosinusitis (EPOS criteria) were subjected for Diagnostic Nasal Endoscopy.

Condition of the Sinonasal cavity was assessed for presence or absence of polyps, state of Sinonasal mucosa, hypertrophy, crusting and type of discharge.

Patient with malignancies, congenital anomalies, autoimmune / granulomatous diseases were excluded from the study.

Depending upon the findings at Diagnostic Nasal Endoscopy patients were segregated into two groups, i.e., Chronic Rhinosinusitis with Polyps (CRSwNP) and Chronic Rhinosinusitis without Polyps (CRSsNP).

All these patients were evaluated according to the standard laboratory profiles of allergy. CT scan of paranasal sinuses were taken and scores were recorded.

All the patients who satisfied EPOS criteria were included in the study after obtaining their consent.

SNOT 22 Sino Nasal Outcome Test Questionnaire, a validated tool was used to assess the patients.

Data analysis and interpretation: Data was entered into Microsoft Excel (Windows 7; Version 2007) and analyses were done using the Statistical Package for Social Sciences (SPSS) for Windows software (version 22.0; SPSS Inc, Chicago).

Descriptive statistics such as mean and standard deviation (SD)for continuous variables, frequencies and percentages were calculated for categorical Variables were determined.

Association between Variables was analyzed by using Chi-Square test for categorical Variables.

Comparison of mean of quantitative variables were analyzed using unpaired $t$ test and Mann-Whitney test after checking the data for normality distribution by using Shapiro-Wilk Test.

Bar charts and Pie charts were used for visual representation of the analyzed data.

Level of significance was set at 0.05 . 


\section{Results}

Table-1: Association between study group and bio-social characteristics ( $\mathrm{N}=\mathbf{2 3 1})$.

\begin{tabular}{|c|c|c|c|}
\hline \multirow[b]{2}{*}{ Parameter } & \multicolumn{2}{|c|}{ Chronic rhinosinusitis } & \multirow[b]{2}{*}{ P Value } \\
\hline & $\begin{array}{l}\text { With polyposis } \\
(n=140) n(\%)\end{array}$ & $\begin{array}{l}\text { Without polyposis } \\
\qquad(\mathrm{n}=91) \mathrm{n}(\%)\end{array}$ & \\
\hline \multicolumn{4}{|l|}{ Age (in years) } \\
\hline$\leq 20$ & $4(2.9)$ & $2(2.2)$ & \multirow{6}{*}{0.248} \\
\hline $21-30$ & $60(42.9)$ & $30(33.0)$ & \\
\hline $31-40$ & $33(23.6)$ & $30(33.0)$ & \\
\hline $41-50$ & $17(12.1)$ & $17(18.7)$ & \\
\hline $51-60$ & $21(15.0)$ & $8(8.8)$ & \\
\hline$>60$ & $5(3.6)$ & $4(4.4)$ & \\
\hline Mean (SD) & $34.7(12.8)$ & $35.9(11.6)$ & \\
\hline \multicolumn{4}{|l|}{ Gender } \\
\hline Male & $71(50.7)$ & $43(47.3)$ & \multirow[t]{2}{*}{0.607} \\
\hline Female & $69(49.3)$ & $48(52.7)$ & \\
\hline Asthma & $29(20.7)$ & $11(12.1)$ & 0.090 \\
\hline Smoking & $30(21.4)$ & $20(22.0)$ & 0.921 \\
\hline \multicolumn{4}{|l|}{ Socio-Economic Status } \\
\hline APL & $82(58.6)$ & $67(73.6)$ & \multirow[t]{2}{*}{$0.019^{*}$} \\
\hline BPL & $58(41.4)$ & $24(26.4)$ & \\
\hline
\end{tabular}

Table-2: Association between study group and CT Scan $(\mathbf{N}=231)$

\begin{tabular}{|c|c|c|c|}
\hline \multirow[b]{2}{*}{ CT Scan Score } & \multicolumn{2}{|c|}{ Chronic Rhinosinusitis } & \multirow[b]{2}{*}{ P Value } \\
\hline & $\begin{array}{l}\text { With Polyposis } \\
(n=140) \text { n (\%) }\end{array}$ & $\begin{array}{l}\text { Without Polyposis } \\
\qquad(\mathrm{n}=91) \text { n (\%) }\end{array}$ & \\
\hline No abnormality & 0 & $65(71.4)$ & \multirow{3}{*}{$<0.001 *$} \\
\hline Partial opacification & $69(49.3)$ & $26(28.6)$ & \\
\hline Complete opacification & $71(50.7)$ & 0 & \\
\hline
\end{tabular}


Table-3: Comparison of SNT 22 between two Study Groups $(\mathrm{N}=231)$

\begin{tabular}{|c|c|c|c|c|}
\hline \multirow[b]{2}{*}{ SNT22 } & \multicolumn{2}{|c|}{ Chronic Rhinosinusitis } & \multirow[b]{2}{*}{ P Value } & \multirow[b]{2}{*}{ Effect Size } \\
\hline & $\begin{array}{c}\text { With Polyposis } \\
(n=140) \text { Mean (SD) }\end{array}$ & $\begin{array}{l}\text { Without Polyposis } \\
(\mathrm{n}=91) \text { Mean (SD) }\end{array}$ & & \\
\hline Need to blow your nose & $3.34(1.08)$ & $1.71(0.87)$ & $<0.001^{*}$ & 0.387 \\
\hline Sneezing & $4.24(0.69)$ & $2.35(0.65)$ & $<0.001 *$ & 0.649 \\
\hline Running nose & $3.96(0.76)$ & $2.96(0.64)$ & $<0.001^{*}$ & 0.316 \\
\hline Cough & $3.92(0.81)$ & $1.15(0.82)$ & $<0.001^{*}$ & 0.733 \\
\hline Nasal secretions going to throat & $3.91(0.77)$ & $2.12(0.99)$ & $<0.001^{*}$ & 0.507 \\
\hline Thickened secretions in the nose & $4.03(0.80)$ & $1.79(0.62)$ & $<0.001 *$ & 0.688 \\
\hline A feeling of full or stuffed & $3.71(0.79)$ & $1.09(0.74)$ & $<0.001^{*}$ & 0.734 \\
\hline Giddiness or vertigo & $3.89(0.81)$ & $0.46(0.56)$ & $<0.001 *$ & 0.843 \\
\hline Earache & $3.77(0.92)$ & $0.97(0.79)$ & $<0.001^{*}$ & 0.712 \\
\hline Facial pain or pressure & $3.56(0.85)$ & $0.79(0.70)$ & $<0.001^{*}$ & 0.741 \\
\hline Difficulty to sleep & $3.67(0.72)$ & $1.19(0.85)$ & $<0.001^{*}$ & 0.711 \\
\hline Wake up in the middle of night & $3.81(0.80)$ & $1.46(0.91)$ & $<0.001 *$ & 0.649 \\
\hline Lack of good Night sleep & $3.49(0.97)$ & $1.73(0.96)$ & $<0.001^{*}$ & 0.442 \\
\hline Wake up tired & $3.76(0.84)$ & $1.93(0.95)$ & $<0.001^{*}$ & 0.503 \\
\hline Fatigued or tired during the day & $3.67(0.96)$ & $2.20(0.85)$ & $<0.001^{*}$ & 0.380 \\
\hline $\begin{array}{l}\text { Low performance in doing the } \\
\text { daily activities }\end{array}$ & $3.19(0.78)$ & $2.10(1.01)$ & $<0.001^{*}$ & 0.269 \\
\hline $\begin{array}{l}\text { Low concentration to do daily } \\
\text { activities }\end{array}$ & $3.54(0.86)$ & $2.44(0.74)$ & $<0.001^{*}$ & 0.299 \\
\hline Frustrated/restless/irritated & $3.41(0.97)$ & $1.70(0.99)$ & $<0.001^{*}$ & 0.422 \\
\hline Sadness & $3.41(1.05)$ & $2.08(2.17)$ & $<0.001^{*}$ & 0.146 \\
\hline A feeling of shame & $3.35(1.03)$ & $1.12(1.03)$ & $<0.001^{*}$ & 0.527 \\
\hline Difficulty to feel smell or taste & $3.64(0.96)$ & $1.25(0.88)$ & $<0.001^{*}$ & 0.611 \\
\hline Blockage/Congestion of nose & $3.67(1.03)$ & $1.90(1.07)$ & $<0.001 *$ & 0.406 \\
\hline
\end{tabular}

231 patients satisfied the EPOS criteria for Chronic Rhinosinusitis of which 140 had Polyposis (CRSwNP) and 91 patients had features of Chronic Rhinosinusitis without polyposis (CRSsNP). Most of these patients had history of Nasal and Skin Allergy which was later confirmed by Skin Prick tests.

Male to female susceptibility to polyposis was not significant (CRSwNP,71 and CRSsNP, 69). Smoking tobacco(cigarettes) was also the most important characteristic amongst patients in both groups (CRSwNP,30and CRSsNP,20). Nasal domain scores were compared between the groups, CRSwNP had higher values for both nasal and extra nasal symptoms (CRSwNP,4 and CRSsNP, 2.1).

Nasal obstruction was the most important symptom that significantly affected both CRSwNP and CRSsNP. Irrespective of pattern of clinical presentation it prevailed in both groups (3.71 vs 1.09; p value 0.001). Post-nasal drip with granular 
pharyngitis was also one of the important finding which was found in both polyposis and nonpolyposis patients $(0.80 \mathrm{vs}$ $0.62 ; \mathrm{p}$ value 0.001$)$ Low performance and concentration during daily activities was more common in polyposis patients. ( 3.19 vs 2.44; $p$ value 0.001 ). Seromucinous Rhinorrhoea ( 4.03 vs 1.79 ; $p$ value 0.001 ), Sneezing ( 4.24 vs 2.35 ; $p$ value 0.001 ), Alteration of Taste and Smell (3.64 vs 1.25; p value 0.001 ) were the other RSOM-31 mean symptom scores which significantly affected the patients` quality of life.

Headache, Facial pain and Pressure were also universally present in both groups which had close impact on patient's symptom pattern. Need to use tissues (3.2 vs 3.4), rubbing nose (3.1 vs 3.5) and eyes, blowing nose (3.1vs 3.8) affected CRSwNP more after than CRSsNP. Frustration due to constant irritation, feeling restless, feeling shameful and sadness which are the symptoms suggesting psychological quality of life were more often seen in CRSwNP although good number of nonpolyposis patients too exhibited these features.

\section{Discussion}

In Chronic Rhinosinusitis there is no predictable pattern of symptoms that clearly differentiate CRSwNP and CRSsNP. The range of scores that are provided in this questionnaire thoughtfully justifies the various tools of analysis that can be adopted for different variables. The number of symptoms in the RSOM- 31 makes it difficult for the patients to comprehend and make it more tedious for overall interpretation and necessitates multivariate analysis so a modified shorter version known as SNOT Sinonasal Outcome test has been used. SNOT has multiple variants like SNOT 20, SNOT 21, SNOT 22 with little difference in their significance. SNOT 22 has been standardised and is employed in the present study

The major symptoms like Nasal obstruction, Loss of appetite, Loss of taste and smell, Rhinorrhoea, Sneezing, Post nasal drip have a positive correlation with Endoscopic and CT Scan findings compared to lesser significant symptoms like Facial pain/Pressure, Shortness of Breath, Bad Breath, Occasional Cough, which are almost universal present in all patients with a symptom score of $<2$,which again necessitates further analysis. Smoking did not show significant affliction in the present study, both polyposis and nonpolyposis pts were equal in number (21.4 vs 22.0;0.921) but all of them had CRS. According to EPOS by Le PT et al., CRS was more common in smokers than in nonsmokers (OR 1.7: 95\% CI 1.6-1.9).

The prevalence of self-reported physician-diagnosed CRS within centres was highly correlated with the prevalence of $\mathrm{EP}^{3} \mathrm{OS}$-diagnosed CRS [2].Bronchial asthma was more commonly found with Nasal polyposis [29 (20.7) vs $11(22.1) ; 0.090]$ although it was the only significant association which was evaluated in the present study. In a Korean study by Kim YS et al., sociodemographic factors, male sex, old age, and heavy stress were significantly related with CRS. Influenza vaccination, septal deviation, and persistent allergic rhinitis were significant personal medical risk factors of CRS. Among these risk factors, persistent/moderate to severe allergic rhinitis was proven to be the most significant risk factor for CRS at the population level [3].

Hopkins, Rudmik, Lund found that CRS patients with polyps had greater improvement than patients with CRS without polyps. The predictive value of the SNOT-22 is similar in the UK cohort. Medically recalcitrant patients with CRS considering surgery should make decisions guided by their preoperative quality-of-life impairment, as measured by the SNOT-22 [4].

Lack of good sleep ( 3.39 vs $1.73 ;<0.001)$, Waking up tired $(3.76$ vs $1.93 ;<0.001)$, Fatigued or tired during the day $(3.67$ vs $2.20 ;<0.001)$ were the most affected parameters in the SNOT 22 in the present study. De conde and Solar concluded in their study on burden and epidemiology of CRS that, a refinement of questionnaire-based surveys coupled with sampling of respondents for accuracy likely provides the most accurate assessment of prevalence. Although CRS is marked by sinonasal symptoms, the most problematic symptoms for patients seem to be the symptoms that affect general-health-related domains. Diminished sleep, productivity, cognition, mood, and fatigue are associated with the decision to elect surgical intervention and are associated with diminished healthy utility values [5].

CT scan findings showed partial opacities $(69,49.3)$ and complete opacities $(71,50.7)$ in CRSwNP although even in CRSsNP partial opacities (26) were observed 65 (70.4) were not having any significant polypoidal changes. In a study by Bhattacharjee et al., SNOT-20 scores ranged from 0 (normal) to 78 (mean, 34). The most commonly reported symptom was fatigue. The CT scores ranged from 0 (normal) to 24 (mean, 4.07). Seventy-five patients (34\%) had normal findings on the 


\section{Original Research Article}

CT scan. When the subset of patients with "positive" or "very positive" CT scans were considered, no significant correlation was observed $(\mathrm{r}=0.12, \mathrm{P}<$ or $=$ 0.16 ). For the 132 patients reporting facial pain, the mean CT score was lower than for patients without facial pain (3.78 vs 4.78 , P. 21). They concluded that, patient-based reports of paranasal sinus symptoms failed to correlate with findings on CT scans; therefore, CT should be reserved for delineating the anatomy and pattern of inflammatory paranasal disease prior to surgical intervention, which is almost similar to the present study findings [6].

Deal and Koutanakis in a study using SNOT 20 found that, Polyps were present in 78 patients with CRS, whereas 123 patients did not have polyps. The average CT score was 18 for the polyp group and 9.5 for the patients without polyps $(\mathrm{P}=0.0000)$. Nonpolyp group SNOT-20 scores were 26.5 preoperatively with improvement to 5.1 at 6 months and 5.0 at 12 months postoperatively (85\% improvement). Polyp group SNOT-20 preoperative scores averaged 32.2 with improvement to 9.2 at 6 months and 9.1 at 12 months postoperatively ( $81 \%$ improvement, $\mathrm{P}=0.003)$. They concluded that, the presence of nasal polyps has a significant negative impact on patients with CRS. Patients with nasal polyps have more severe symptoms with less improvement after operative intervention, higher CT scores at presentation, and a significantly higher need for revision surgery [7].

Lund and Kennedy who have standardised the staging of Rhinosinusitis conclude that, with computed tomographic scanning it is possible to more accurately determine the extent of the pathologic condition in rhinosinusitis, a disease in which the severity of symptoms and the appearances on nasal endoscopy have a significantly more unpredictable correlation with the extent of disease. One goal of the Task Force on Rhinosinusitis of the American Academy of Otolaryngology-Head and Neck Surgery was to recommend a system for outcomes research that combines quantification with ease of application. So a combined comprehensive module which includes symptom scores and domains, endoscopic and radiologic findings along with preoperative and postoperative implications is necessary to assess the severity of Rhinosinusitis at different stages of the disease [8].

Bradley and Koutanakis implied that, Preoperative CT scores did not correlate with preoperative symptom scores $(r=0.314)$. The SNOT-20 symptom scores improved $72 \%, 75 \%$, and $77 \%$ at 3-, 6-, and $12-$ month follow-up, respectively, from preoperative values. The severity of rhinosinusitis on preoperative computed tomography scan does not predict the severity of symptoms as assessed by the SNOT-20 inventory in patients undergoing functional endoscopic sinus surgery [9].

Low performance during daily activities (3.19 vs $2.1 ;<0.001)$ and Low concentration in routine activities (3.35 vs $2.44 ;<0.001)$ decreased the quality of daytime activities. Feeling frustrated, restless, irritated (3.41 vs $1.70 ;<0.001)$ and sadness (3.41 vs $2.08 ;<0.001)$, feeling a sense of shame $(3.55$ vs $1.12 ; 0.001)$ were the commonly present affecting their psyche significantly. Craig, Ferguson, Krouse found that, Self-reported chronic symptoms of rhinitis were significantly related to excessive daytime sleepiness and not feeling rested regardless of the amount of sleep. Sleep-disordered breathing, including habitual snoring, is related to hypersomnolence; therefore, associations between rhinitis and sleepiness may be explained by sleepdisordered breathing [10].

In a prospective RCT by Raghab and Lund, both the medical and surgical treatment of CRS significantly improved almost all the parameters of SNOT and SF-36 $(p<0.05)$, with no significant difference being found between the medical and surgical group. The presence of nasal polyps does not imply any negative effect on the quality of life after CRS therapy, either medical or surgical [11].

Decreased sense of smell and taste (3.64 vs $1.25 ;<0.001)$ and Nasal blockade and congestion (3.67 vs $1.90 ;<0.001$ ) were found in CRSwNP and were the most significant Nasal symptoms.

\section{Conclusion}

Symptom patterns in patients with Allergic Rhinosinusitis needs to be analysed and correlated with CRSwNP and CRSsNP. Despite the pathophysiological differences in CRSwNP and CRSsNP there is a considerable overlap in the presentation and no actual correlation can be made by using only symptomatology as a tool. Endoscopic and CT scan findings are the most important entities that finally has to be correlated with symptoms to finally differentiate these two conditions along with immunological studies.

\section{What the study adds to the existing knowledge?}

The present study strengthens the need to evaluate the symptoms with relation to endoscopic and imaging 


\section{Original Research Article}

studies in Chronic Rhinosinusitis as justified by the literature. To achieve this goal, it necessitates to device a comprehensive module of evaluation which can serve to congregate clinical symptoms, endoscopy and imaging studies under one single protocol which will simplify the management effectively thereafter.

\section{Author's contributions}

Dr. Nagavara Kalegowda Balaji: Concept, study design and analysis

Dr. Ravi D.: Concept, study design and analysis.

Dr. MH Prasad: Concept, study design and analysis

Funding: Nil, Conflict of interest: Nil

Permission from IRB: Yes

\section{References}

1. Fokkens WJ, Lund VJ, Mullol J, Bachert C, Alobid I, Baroody F, et al. European Position Paper on Rhinosinusitis and Nasal Polyps 2012. Rhinol. 2012; 50(1): 1-12. doi: 10.4193/Rhino50E2.

2. Hastan D, Fokkens WJ, Bachert C, Newson RB, Bislimovska J, Bockelbrink A, et al. Chronic rhinosinusitis in Europe--an underestimated disease. A GA²LEN study. Allergy. 2011;66(9):1216-1223. doi: 10.1111/j.1398-9995.2011.02646.x. Epub 2011 May 24.

3. Kim YS, Kim NH, Seong SY, Kim KR, Lee GB, Kim KS. Prevalence and risk factors of chronic rhinosinusitis in Korea. Am J Rhinol Allergy. 2011; 25(3): 117-121. doi: 10.2500/ajra.2011.25.3630.

4. Hopkins C, Rudmik L, Lund VJ. The predictive value of the preoperative Sinonasal Outcome Test-22 score in patients undergoing endoscopic sinus surgery for chronic rhinosinusitis.Laryngoscope. 2015;125(8): 1779 -1784. doi: 10.1002/lary.25318. Epub 2015 Apr 17.

5.DeConde AS, Soler ZM. Chronic rhinosinusitis: Epidemiology and burden of disease. Am J Rhinol Allergy. 2016;30(2):134-139. doi: 10.2500/ajra.2016. 30.4297.
6. Bhattacharyya T, Piccirillo J, Wippold FJ. Relationship between patient-based descriptions of sinusitis and paranasal sinus computed tomographic findings. Arch Otolaryngol-Head Neck Surg. 1997; 123 (11): 1189-1192.

7. Deal RT, Kountakis SE. Significance of nasal polyps in chronic rhinosinusitis: symptoms and surgical outcomes. Laryngo. 2004;114(11):1932-1935. doi:10. 1097/ 01.mlg. 0000147922.12228.1f

8. Lund VJ, Kennedy DW. Staging for rhinosinusitis. Otolaryngol Head Neck Surg. 1997;117(3 Pt 2):S35S40. doi:10.1016/S0194-59989770005-6

9. Bradley DT, Kountakis SE. Correlation between computed tomography scores and symptomatic improvement after endoscopic sinus surgery. Laryngo. 2005;115(3):466-469.doi:10.1097/01.mlg.0000157840. 55659.62

10.Craig TJ, Ferguson BJ, Krouse JH. Sleep impairment in allergic rhinitis, rhinosinusitis, and nasal polyposis. Am J Otolaryngol. 2008;29(3):209-217. doi: 10.1016/j. amjoto.2007.06.002.

11.Ragab SM, Lund VJ, Scadding G, Saleh HA, Khalifa MA. Impact of chronic rhinosinusitis therapy on quality of life: a prospective randomized controlled trial. Rhinol. 2010;48(3):305-311. doi: 10.4193/Rhin08.137.

12. Sobol SE, Wright ED, Frenkiel S. One-year outcome analysis of functional endoscopic sinus surgery for chronic sinusitis. J Otolaryngol-Head Neck Surg. 1998;27(5):252-257.

13.Soler ZM, Mace J, Smith TL.Symptom-based presentation of chronic rhinosinusitis and symptom-specific outcomes after endoscopic sinus surgery. Am J Rhinol. 2008;22(3):297-301. doi: 10.2500/ajr.2008. 22. 3172.

14. Abdalla S, Alreefy H, Hopkins C. Prevalence of sinonasal outcome test (SNOT-22) symptoms in patients undergoing surgery for chronic rhinosinusitis in the England and Wales National prospective audit. Clin Otolaryngol. 2012;37(4):276-282. doi: 10.1111/j.17494486. 2012.02527.x.

\section{How to cite this article?}

Balaji N.K, Ravi D, Prasad MH. Assessment of symptom severity in chronic rhinosinusitis with and without polyps- a prospective study in tertiary care centre at Mandya. Trop J Ophthalmol Otolaryngol.2019;4 (4):270276.doi:10.17511/jooo.2019.i04.03 